\title{
PERFECT OPEN AND DISTINGUISHABLE MULTIVALUED MAPS
}

BY

\author{
ERIC JOHN BRAUDE
}

\begin{abstract}
It is shown that perfect open multivalued maps preserve Z-analytic sets (which include compact zero sets) as well as other objects of descriptive set theory. The concept of "distinguishability", introduced by Frolík, is applied to multivalued maps, yielding a new class of such maps with similar preservation properties.

That the projection of a compact zero set is a zero set is one corollary, and another is a generalized $\mathcal{S}_{\delta}$ diagonal metrization theorem.
\end{abstract}

The notion of a descriptive $Z$-analytic set (abbreviated to "Z-analytic set" in this paper) was introduced and studied in [2]. Whereas invariance under continuous maps is a salient feature of analytic sets, Z-analytic sets do not have this property. We present here two types of multivalued maps which preserve $Z$-analytic sets. The study of each leads to results on projection and metrization.

In $\$ 2$ we establish the invariance of $Z$-analytic sets under perfect open maps (Corollary 2.5). Examples 2.2 and 2.3 illustrate the failure of weaker conditions to preserve even compact zero sets. The general result underlying this section is Theorem 2.4, and the final Corollary (2.8) states that the projection of a compact zero set is a zero set.

In $\$ 3$, so-called "graph-distinguishable" multivalued maps are introduced and studied. With suitable restrictions, they too preserve $\mathcal{E}_{\text {analytic sets }}$ (Corollary 3.10). Using graph-distinguishability, a generalization is established of the theorem which asserts that compact spaces with $\mathcal{G}_{\delta}$ diagonal are metrizable.

The author is indebted to Herman Kahn and John Mack for suggesting improvements on his results in [3] which have led to Theorem 2.4 (i) and its proof.

1. Definitions and preliminaries.

1.1. All spaces will be taken to be Hausdorff. (As in [2], this assumption can be dropped for many of the results established below.)

Received by the editors May 15, 1972 and, in revised form, November 27, 1972. AMS (MOS) subject classifications (1970). Primary 54C50, 54C60, 54C10, 54H05, 04A15; Secondary 54E35, 54E 45, 54G20.

Key words and phrases. Perfect, open maps, distinguishable, multivalued maps, $z$ analytic sets, descriptive set theory, $\bigotimes_{\delta}$ diagonal, projection, Baire, zero sets, Borel sets, analytic sets, Souslin. 
If $(X, \mathfrak{J})$ is a topological space, then $C^{*}(X, \mathfrak{J})$ (or, when the topology is self-evident, $\left.C^{*}(X)\right)$ denotes the space of bounded continuous real-valued functions on $X$ with the supremum metric.

The zero sets of $X$ are the sets of the form $f^{-1}(0)$ (denoted $\left.Z(f)\right), f$ an element of $C^{*}(X)$.

If $X$ and $Y$ are topological spaces, $C(X, Y)$ is the space of continuous functions of $X$ into $Y$ with the compact open topology.

1.2. A multivalued map is a triple, $F: X \rightarrow Y$, where $F \subseteq X \times Y$. The graph of $F: X \rightarrow Y$ is $F$. For $A \subseteq X, F(A)$ denotes $\{y \in Y: F \cap(A \times\{y\}) \neq \varnothing\}$. For $B \subseteq Y, F^{-1}(B)$ denotes $\{x \in X: F \cap(\{x\} \times B) \neq \varnothing\}$. A multivalued map $F: X \rightarrow$ $Y$ is called u.s.c. (upper semicontinuous) if $F^{-1}(B)$ is closed for every closed $B$ in $Y$; disjoint if $F\left(x_{1}\right) \cap F\left(x_{2}\right)=\varnothing$ for $x_{1} \neq x_{2}(F(x)$ means $F(\{x\}))$; and continuous if it is u.s.c. and $F^{-1}(B)$ is open for every open $B$ in $Y$. We call $F: X \rightarrow Y$ open (closed) if $F(A)$ is open (closed) for every open (closed) $A \subseteq X$; range-compact (domain-compact) if $F(x)\left(F^{-1}(y)\right)$ is compact for every $x$ in $X$ ( $y$ in $Y$ ); range-perfect (domain-perfect) if it is closed, range compact and u.s.c. (closed, domain-compact and u.s.c.); and perfect if it is both range-perfect and domain-perfect.

If $\mathcal{Q}$ is a family of subsets of $X$ and $F: X \rightarrow Y$ is a correspondence, then $F(\mathfrak{Q})$ denotes $\{F(A): A \in \mathbb{Q}\}$.

If $F: X \rightarrow Y$ and $G: Y \rightarrow Z$ are correspondences, then $G \circ F$ is defined to be $\{(x, z) \in X \times Z: z \in G(F(x))\}$.

1.3. By $\mathcal{C}(X)$ we denote the space of compact subsets of $X$ with the exponential topology. Then $\mathcal{C}(X)$ is metrizable or separable if $X$ is.

1.4. Let $Z(X)$ denote the class of zero sets of $X$. The Baire sets of $X$ are precisely the members of the smallest class of subsets containing $Z(X)$ and closed under countable unions and countable intersections.

1.5. Let $\mathbf{N}$ denote the set of (strictly) positive integers with the discrete topology. Let $\mathbf{I}=\mathbf{N}^{\mathbf{N}}$ with the product topology. For each element $\mathbf{i}=$ $\left(i_{1}, i_{2}, i_{3}, \ldots\right)$ of $\mathbf{I}$ and each $n$ in $\mathbf{N}, \mathbf{i} \mid n$ denotes the finite sequence $\left(i_{1}, i_{2}, \ldots, i_{n}\right)$. The $Z$-Souslin sets of $X$ are precisely the sets of the form $\bigcup_{i \in I} \bigcap_{n=1}^{\infty} Z(i \mid n)$, where $Z(i \mid n)$ is a zero set for every finite sequence $i \mid n$. It is easy to show that every Baire set is $Z$-Souslin.

1.6. A Z -analytic subset of $X$ is a set of the form $K(I)$ where $K: I \rightarrow X$ is a range-compact u.s.c. multivalued map for which there exists a continuous map $F: \mathbb{I} \rightarrow C^{*}(X)$ such that $K(\mathbf{i})=Z(F(i))$ for each $\mathbf{i}$ in $\mathbf{I}$. If, in addition, $K\left(i_{1}\right) \cap K\left(i_{2}\right)=\varnothing$ for $i_{1} \neq i_{2}$, then $K(I)$ is termed descriptive Baire in $X$.

1.7. A subset $A$ of $X$ is distinguishable in $X$ if there exists a separable metrizable space $M$ and a continuous single-valued function $q$ of $X$ into $M$ such 
that $q(A) \cap q(X \sim A)=\varnothing$. A family $\mathcal{H}$ of subsets of $X$ is distinguisbable if there exists a separable metrizable space $M$ and a continuous function $q$ of $X$ into $M$ which distinguishes every element of $\mathcal{H}$ (see [2]). We say that the pair $(q, M)$ distinguishes $A$ or $\mathcal{H}$.

The following properties of distinguishable sets and families will be used in the sequel.

(i) If, for $n=1,2, \ldots$, the family $\mathcal{H}_{n}$ is distinguished by $\left(q_{n}, M_{n}\right)$, then $\bigcup_{n=1}^{\infty} \mathcal{H}_{n}$ is distinguished by $\left(\Pi_{n=1}^{\infty} q_{n}, \Pi_{n=1}^{\infty} M_{n}\right)$ where $\Pi_{n=1}^{\infty} q_{n}$ is defined by $\Pi_{n=1}^{\infty} q_{n}(x)=\left(q_{1}(x), q_{2}(x), \ldots\right)$.

(ii) If $(q, M)$ distinguishes a family $\mathcal{H}$, then it also distinguishes every family whose elements are unions or intersections of elements of $\mathcal{H}$.

Since zero sets are distinguishable, so are $\mathcal{Z}$ Souslin sets by (i) and (ii).

1.8. Lemma. Compact distinguisbable sets are zero sets [5, p. 409].

1.9. Lemma. Z-analytic sets are Z-Souslin sets and descriptive Baire sets are Baire sets (see [2]).

1.10. Lemma. A subset $K(\mathrm{I})$ of $X$, for $K: \mathrm{I} \rightarrow X$ u.s.c. and range-compact, is $\mathcal{Z}$-analytic if, and only if, $\{K(\mathbf{i}): \mathbf{i} \in \mathbf{I}\}$ is distinguishable [2, p. 412].

1.11. We say that a multivalued map $F: X \rightarrow Y$ preserves property $\mathrm{P}$ if $F(A)$ has property $\mathrm{P}$ as a subspace of $F(X)$ whenever $A$ has property $\mathrm{P}$ in $X$.

2. Perfect open multivalued maps.

2.1. Example 2.2 shows that continuous open closed single-valued maps between normal Lindelöf spaces generally preserve neither zero sets, Z-Souslin sets, distinguishable sets nor $\mathcal{Z}$-analytic sets, even if these sets are also compact. Example 2.3 illustrates the same conclusion for perfect maps-in fact, for continuous single-valued maps with compact domain.

2.2. Example. Let $P=[0,1]$, and let $\mathcal{T}$ be the topology on $P$ whose basis is given by

$$
\{\{x\}: 0<x \leq 1\} \cup\{H \subseteq P: 0 \in H \text { and } P \sim H \text { is countable }\} .
$$

Then $(P, \mathcal{J})$ is a normal Lindelöf Hausdorff space.

Let $B=\{0,0)\} \cup[\{1 / n: n \in \mathbf{N}\} \times P]$, and let $\mathcal{S}$ be the topology on $B$ with basis

$$
\left\{\{1 / n\} \times T_{n}: n \in \mathbf{N} \text { and } T_{n} \in \mathcal{T}\right\} \cup \mathcal{H},
$$

where the family $\mathcal{H}$ consists of sets of the form

$$
\{(0,0)\} \cup \bigcup_{n=1}^{\infty}\left[\left\{\frac{1}{n}\right\} \times U_{n}\right],
$$


where $U_{n} \in \mathcal{T}$ and there exists $m \in \mathbf{N}$ for which $n \geq m$ implies $0 \in U_{n}$.

Thus, the closed sets of $(B, \delta)$ consist of: (i) all subsets of the form $\{(0,0)\} \cup \bigcup_{n=1}^{\infty}\left[\{1 / n\} \times L_{n}\right]$ where $L_{n} \subseteq P$ and $P \sim L_{n} \in \mathcal{T}$; and (ii) all subsets of the form $K=\bigcup_{n=1}^{\infty}\left[\{1 / n\} \times V_{n}\right]$ where $V_{n} \subseteq P, P \sim V_{n} \in \mathcal{T}$, and there exists $m$ in $\mathbf{N}$ for which $n \geq m$ implies $0 \not k V_{n}$. Hence $(B, \mathcal{S})$ is a normal Lindelö Hausdorff space.

Define $f:(B, \mathcal{S}) \rightarrow(P, \mathcal{T})$ by $f(0,0)=0$ and $f(1 / n, x)=x$. The function $f$ is continuous and open. It is also closed. (The image of a closed set of type (i) is clearly closed, and the same is true for sets of type (ii) whenever $0 \in V_{n}$ for some $n$. If $0 \notin V_{n}$ for all $n$ in $\mathbf{N}$, then $V_{n}$ is countable for all $n$, and $f[K]=$ $\bigcup_{n=1}^{\infty} f\left[\{1 / n\} \times V_{n}\right]$ is a countable subset of $(0,1]$ and is thus closed in $(P, \mathcal{T})$.)

Now $\{(0,0)\}=Z(g)$ where $g$ is the continuous function of $B$ into $R$ defined by $g(0,0)=0$ and $g(1 / n, x)=1 / n$. Thus, $\{(0,0)\}$ is a compact zero set of $(B, \mathcal{S})$ but $f[\{(0,0)\}]=\{0\}$ is not a zero set of $(P, \mathcal{J})$. In $(B, \mathcal{S})$, the set $\{(0,0)\}$ is also $\mathcal{Z}$-analytic (therefore $\mathcal{Z}$-Souslin and distinguishable). However, $\{0\}$ is not even distinguishable in $(P, \mathfrak{J})$, for otherwise it would be a zero set by Lemma 1.8.

2.3. Example. We use the same symbols as above. Let $\mathcal{U}$ be the topology on $P$ whose basis is

$\{\{x\}: 0<x \leq 1\} \cup\{H \subseteq P: 0 \in H$ and $P \sim H$ is finite $\}$.

Then $(P, \mathcal{U})$ is a compact Hausdorff space.

Let $F_{n}=\{1 / n\} \times[0,1 / n]$ and let $G=\{(0,0)\} \cup \cup_{n=1}^{\infty} F_{n}$. Let $\mathcal{O}$ be the topology on $G$ with basis

$$
\left\{\{(0,0)\} \cup \bigcup_{n=m}^{\infty} F_{n}: m \in \mathbf{N}\right\} \cup\left\{\left\{\frac{1}{n}\right\} \times U: n \in \mathbf{N}, U \subseteq\left[0, \frac{1}{n}\right] \text {, and } U \in \mathcal{U}\right\} \text {. }
$$

Then $(G, \mathcal{O})$ is a compact Hausdorff space.

The function $f:\left(G, C^{O}\right) \rightarrow(P, \mathcal{U})$, defined by $f(x, y)=y$, is continuous; but $f(\{(0,0)\})=\{0\}$ is not a zero set of $(P, \mathcal{U})$, even though $\{(0,0)\}=Z(b)$ where $b$ is the element of $C^{*}(G, \mathcal{C})$ defined by $b(x, y)=x$.

2.4. Theorem. Perfect open single-valued maps (more generally, open, closed, domain-compact multivalued maps) preserve (i) zero sets, (ii) $\mathfrak{Z}$-Souslin sets, (iii) distinguis bable families.

Proof. Let $F: X \rightarrow Y$ be an open closed domain-compact multivalued map with $F(X)=Y$.

(i) Let $A=Z(g)$ for $g \in C^{*}(X)$. We can assume that $g$ is positive valued. Define the real-valued map $b$ by

$$
b(y)=\inf \left\{g(x): x \in F^{-1}(y)\right\} .
$$


The continuity of $b$ is proved as follows: Given $\epsilon^{\prime}>0$, we have $y_{0} \in A\left(y_{0}\right)$ for each $y_{0}$ in $Y$ where

$$
A\left(y_{0}\right)=F\left[g^{-1}\left(\left\{r:\left|b\left(y_{0}\right)-r\right|<\epsilon\right\}\right)\right] \cap\left[F\left[g^{-1}\left(\left\{r: r \leq b\left(y_{0}\right)-\epsilon\right\}\right)\right]\right]^{\prime} .
$$

(For $L \subseteq Y, L^{\prime}$ denotes $Y \sim L_{\text {. }}$ ) Since $F: X \rightarrow Y$ is open and closed, $A\left(y_{0}\right)$ is open. For every element $y$ of $A\left(y_{0}\right)$, we have $\left|b(y)-b\left(y_{0}\right)\right|<\epsilon$.

Since $F: X \rightarrow Y$ is domain-compact, $b(y) \in g\left[F^{-1}(y)\right]$ for each $y$ in $Y$; so $y \in Z(b)$ if, and only if, $Z(g) \cap F^{-1}(y) \neq \varnothing$. Hence $F(Z(g))=Z(b)$ is a zero set.

(ii) Let $A=\bigcup_{\mathrm{i} \in \mathrm{I}} \bigcap_{n=1}^{\infty} A(\mathrm{i} \mid n)$, where $A(\mathrm{i} \mid n)$ is a zero set for each $\mathrm{i} \mid n$. It can be assumed that $A(\mathrm{i} \mid n) \supseteq A(\mathrm{i} \mid n+1)$ for every $\mathrm{i}$ in $\mathrm{I}$ and $n$ in $\mathbf{N}$.

We prove that $F\left[\bigcap_{n=1}^{\infty} A(\mathrm{i} \mid n)\right]=\bigcap_{n=1}^{\infty} F[A(\mathrm{i} \mid n)]$ for each $\mathrm{i} \in \mathrm{I}$ as follows. If $y \in \bigcap_{n=1}^{\infty} F[A(\mathrm{i} \mid n)]$, then for each $n$ in $\mathrm{N}, y \in F\left(z_{n}\right)$ for some $z_{n}$ in $A(\mathrm{i} \mid n)$. Since $F^{-1}(y)$ is compact, it contains a limit point, $z_{0}$, of $\left\{z_{n}\right\}_{n=1}^{\infty}$. Now $z_{0} \epsilon$ $\bigcap_{n=1}^{\infty} A(\mathrm{i} \mid n)$ for otherwise $z_{0} \in[A(\mathrm{i} \mid m)]^{\prime}$ for some $m$, which is impossible because the open set $[A(i \mid m)]^{\prime}$ does not contain $z_{n}$ for $n \geq m$. Thus,

$$
y \in F\left(z_{0}\right) \subseteq F\left[\bigcap_{n=1}^{\infty} A(\mathrm{i} \mid n)\right] .
$$

Since $F\left(\bigcap_{n=1}^{\infty} A(\mathrm{i} \mid n)\right] \subseteq \bigcap_{n=1}^{\infty} F[A(\mathrm{i} \mid n)]$, we have

$$
F(A)=\bigcup_{i \in \mathrm{I}} F\left[\bigcap_{n=1}^{\infty} A(i \mid n)\right]=\bigcup_{\mathrm{i} \in \mathrm{I}} \bigcap_{n=1}^{\infty} F[A(\mathrm{i} \mid n)],
$$

which is a $\mathcal{Z}$-Souslin set because, by part (i), $F[A(i \mid n)]$ is a zero set for every $i \mid n$

(iii) Let $\mathcal{F}$ be a family of subsets of $X$ distinguished by $(q, M)$. Define the function $p$ of $Y$ into $\mathcal{C}(M)$ by $p(y)=q\left[F^{-1}(y)\right]$.

The continuity of $p$ is established as follows. First let $H$ be an open set in $M$ containing $q\left[F^{-1}\left(y_{0}\right)\right]$. Then $y_{0}$ belongs to the open set $V=\left[F\left(\left[q^{-1}(U)\right]^{\prime}\right)\right]^{\prime}$, and $y \in V$ implies $y \notin F(x)$ for all $x$ in $\left[q^{-1}(U)\right]^{\prime}$.

Thus, for every $y$ in $V$ we have $F^{-1}(y) \subseteq q^{-1}(U)$, and so

$$
p(y)=q\left[F^{-1}(y)\right] \subseteq U .
$$

Now let $W$ be an open set in $M$ with $W \cap q\left[F^{-1}\left(y_{0}\right)\right] \neq \varnothing$. For $y$ belonging to the open set $F\left[q^{-1}(W)\right]$, we have $F^{-1}(y) \cap q^{-1}(W) \neq \varnothing$, i.e. $W \cap q\left[F^{-1}(y)\right] \neq \varnothing$.

We now show that $(p, \mathcal{C}(M))$ distinguishes $F(\mathfrak{F})$. If $U \in \mathcal{F}, y \in F(H)$ and $p\left(y^{\prime}\right)=p(y)$ for some $y^{\prime}$ in $Y$, then $y \in F(x)$ for some $x$ in $H$, and $q\left[F^{-1}\left(y^{\prime}\right)\right]=$ $q\left[F^{-1}(y)\right]$. Hence, there is an element $x^{\prime}$ of $F^{-1}\left(y^{\prime}\right)$ for which $q\left(x^{\prime}\right)=q(x)$. Since $(q, M)$ distinguishes $H$, we have $x^{\prime} \in H$, and so $y^{\prime} \in F\left(x^{\prime}\right) \subseteq F(H)$. Thus $(P, \mathcal{C}(M)$ distinguishes $F(\mathcal{F})$.

2.5. Corollary. Z-analytic sets are preserved by perfect open multivalued 
maps, and descriptive Baire sets by disjoint sucb maps.

Proof. Let $A$ be a $\mathscr{Z}$-analytic set in $X$ with the representation $A=K(I)$ of 1.6. Thus $\{K(\mathbf{i}): \mathbf{i} \in I\}$ is distinguishable (1.10). Let $F: X \rightarrow Y$ be open and perfect. By [5, p. 414], $F \circ K$ is u.s.c. and range-compact. By part (iii) of Theorem 2.4, $\{F \circ K(\mathbf{i}): \mathbf{i} \in \mathbf{I}\}$ is distinguishable. Hence $F(A)=F \circ K(\mathrm{I})$ is $\mathcal{Z}$ analytic in $Y$ by (1.10).

The argument for descriptive Baire sets is similar.

2.6. Remark. When the underlying space is compact, the family of $\mathfrak{Z}$-analytic sets coincides with the family of $\mathcal{Z}$-Souslin sets [2, p. 423]. Jayne [6] has demonstrated additional points of analogy in this case with the classical theory of Borel and analytic sets in complete separable metric spaces.

Corollary 2.7 shows that if we restrict ourselves to projections in product spaces, the behaviour of $\mathcal{Z}$-Souslin sets in compact sets remains consistent with the classical theory.

2.7. Corollary. The projection of a Z.Souslin set in a product of compact spaces is Z.Souslin.

Proof. This follows from Corollary 2.5 since projection here is perfect and open.

Finally, we have the following result, announced in [3], which follows directly from Theorem 2.4, part (i).

2.8. Corollary. The projection of a compact zero set is a zero set. (More generally, projection parallel to compact factors preserves zero sets.)

3. Graph-distinguishable multivalued maps.

3.1. Definition. A multivalued map $F: X \rightarrow Y$ will be called graph-distinguishable if $F$ is a distinguishable subset (see 1.7) of $X \times Y$.

3.2. Example. Multivalued maps (in fact, even continuous single-valued maps) which are graph-distinguishable, do not generally preserve distinguishable sets.

Let $\mathcal{U}$ be a normal Hausdorff topology on the unit interval $P$, in which every singleton is a $\varrho_{\delta}$, and which contains a compact subset $S$ which is not a $\varrho_{\delta}$ (e.g. the compact space $A_{2}$ of Alexandroff and Urysohn [1]). Let $\mathfrak{D}$ be the discrete topology on $P$, and let $\Delta=\{(x, x): x \in P\}$.

For each $x$ in $P$, let $\{x\}=\bigcap_{n=1}^{\infty} U(n, x)$ where $U(n, x) \in \mathcal{U}$ and $n \in \mathrm{N}$. Then

$$
\Delta=\bigcap_{n=1}^{\infty}[\bigcup\{U(n, x) \times\{x\}: x \in P\}] \text {. }
$$


Since $\Delta$ is a closed $\mathcal{G}_{\delta}$ of the normal space $(P, \mathcal{U}) \times(P, \mathscr{D})$, it is a zero set, and so the continuous function $\Delta:(P, \mathscr{D}) \rightarrow(P, \mathcal{U})$ is graph-distinguishable.

Now $S$ is distinguishable in $(P, \mathscr{D})$, but $\Delta(S)=S$ is not distinguishable in $(P, \mathcal{U})$ otherwise it would be a $\oint_{\delta}$ by Lemma 1.8 .

3.3. If, however, a distinguishable set is compact (and therefore a zero set by Lemma 1.8 ), then it is preserved by continuous graph-distinguishable maps. This is a consequence of the following theorem.

3.4. Theorem. Graph-distinguishable u.s.c. range-compact multivalued maps preserve compact zero sets.

Proof. Let $F: X \rightarrow Y$ be range-compact and graph-distinguishable, and let $K$ be a compact zero set of $X$. We denote by $P_{X}\left(P_{Y}\right)$, the projection of $X \times Y$ onto $X(Y)$.

If $K=Z(q)$ for $q \in C^{*}(X)$, then $P \bar{X}^{-1}(K)=Z(\bar{q})$, where $\bar{q}$ is that element of $C^{*}(X \times Y)$ defined by $\bar{q}(x, y)=q(x)$. Thus $F \cap P_{X}^{-1}(K)$ is distinguished in $X \times Y$.

Since $F: X \rightarrow Y$ is u.s.c. and range-compact, $F$ is closed in $X \times Y$ and $F(K)$ is compact (see [5, pp. 414,415]). Thus $F \cap P_{X}^{-1}(K)=F \cap[K \times F(K)]$ is compact.

It follows from Lemma 1.8 that the compact set $F \cap P_{X}^{-1}(K)$ is a zero set. Hence $F(K)=P_{Y}\left[F \cap P_{X}^{-1}(K)\right]$ is a zero set by Corollary 2.8.

3.5. Remarks. The graph-distinguishability of $F: X \rightarrow Y$ implies the distinguishability of each of the sets $F(x)$ and $F^{-1}(y), x \in X$ and $y \in Y$. [For if $(q, M)$ distinguishes $F$ in $X \times Y$, then $\left(q_{x}, M\right)$ distinguishes $F(x)$ in $Y$, where $q_{x}$ is defined by $q_{x}(y)=q(x, y)$.]

The converse is not true in general. Let $X$ be a nonmetrizable first countable compact Hausdorff space (e.g. the space $A_{2}$ of [1]), and let $\Delta$ be the diagonal $\{(x, x): x \in X\}$ in $X \times X$. Then $\Delta(x)$ and $\Delta^{-1}(x)$ are distinguishable for each $x$ in $X$, but if $\Delta: X \rightarrow X$ were graph-distinguishable, $\Delta$ would be a $\mathcal{G}_{\delta}$ by Lemma 1.8 , and so $X$ would be metrizable by the well-known metrization theorem (Corollary 3.17 below). We demonstrate in part (i) of Theorem 3.7 that it is the distinguishability of the families $\{F(x): x \in X\}$ and $\left\{F^{-1}(y): y \in Y\right\}$ which supplies a sufficient condition for $F$ to be graph-distinguishable.

The graph-distinguishability of $F: X \rightarrow Y$, however, does not generally imply the distinguishability of the families $\{F(x): x \in X\}$ and $\left\{F^{-1}(y): y \in Y\right\}$. (From Example 3.2, $\Delta:(P, \mathfrak{T}) \rightarrow(P, \mathcal{U})$ is a graph-distinguishable map, but if $\{\Delta(p): p \in P\}$ were distinguished by $(g, M)$ (say), then the compact nonmetrizable space $(P, \mathcal{U})$ would be homeomorphic to the metrizable space $M$.$) It is for this$ reason that the separability condition is introduced below. 
3.6. Lemma. If $F: X \rightarrow Y$ is a graph-distinguishable multivalued map and $X$ is separable, then $\{F(x): x \in X\}$ is distinguishable.

Proof. Suppose that $(q, M)$ distinguishes $F \subseteq X \times Y$, and $\left\{x_{n}\right\}_{n=1}^{\infty}$ is dense in $X$. Define $q_{n}: Y \rightarrow M$ by $q_{n}(y)=q\left(x_{n}, y\right)$.

We prove that $\left(\Pi_{n=1}^{\infty} q_{n}, M^{\mathrm{N}}\right)$ distinguishes $\{F(x): x \in X\}$.

Let $y \in F(x)$ and let $y^{\prime}$ be an element of $Y$ for which

$$
\left(\prod_{n=1}^{\infty} q_{n}\right)\left(y^{\prime}\right)=\left(\prod_{n=1}^{\infty} q_{n}\right)(y) .
$$

Thus $q\left(x_{n}, y^{\prime}\right)=q\left(x_{n}, y\right)$ for all $n$ in $\mathbf{N}$.

Let $\mathscr{D}$ be the set of open neighborhoods of $x$. Thus $(\mathscr{D}, \subseteq)$ is a directed set (see, for example, $\left[7\right.$, p. 65]). For each $D$ in $\mathcal{D}$, choose $x_{n(D)} \in D \cap$ $\left\{x_{n}: n \in \mathbf{N}\right\}$. Let $S:(D, \subseteq) \rightarrow X \times Y$ and $T:(D, \subseteq) \rightarrow X \times Y$ be the nets defined by $S(D)=\left(x_{n(D)}, y\right)$ and $T(D)=\left(x_{n(D)}, y^{\prime}\right)$. Thus, $S$ and $T$ converge to $(x, y)$ and $\left(x, y^{\prime}\right)$ respectively.

Since $q: X \times Y \rightarrow M$ is continuous, $q \circ S$ and $q \circ T$ converge to $q(x, y)$ and $q\left(x, y^{\prime}\right)$ respectively. However, for every $D$ in $\mathcal{D}$ we have

$$
q \circ S(D)=q\left(x_{n(D)}, y\right)=q\left(x_{n(D)}, y^{\prime}\right)=q \circ T(D) .
$$

Thus $q \circ S=q \circ T$, and since $M$ is Hausdorff, $q(x, y)=q\left(x, y^{\prime}\right)$.

Since $(x, y) \in F$ and $(q, M)$ distinguishes $F$, we have $\left(x, y^{\prime}\right) \in F$, and hence $y^{\prime} \in F(x)$.

3.7. Theorem. Let $F: X \rightarrow Y$ be a multivalued map.

(i) If $\{F(x): x \in X\}$ and $\left\{F^{-1}(y): y \in Y\right\}$ are distinguisbable, then $F: X \rightarrow$ $Y$ is graph-distinguishable.

(ii) The converse to (i) bolds if each of $X$ and $Y$ is either separable or compact.

Proof. (i) Suppose that $\left(q_{X}, M_{X}\right)$ distinguishes $\left\{F^{-1}(y): y \in Y\right\}$ and $\left(q_{Y}, M_{Y}\right)$ distinguishes $\{F(x): x \in X\}$.

We prove that $\left(q_{X} \times q_{Y}, M_{X} \times M_{Y}\right)$ distinguishes $F \subseteq X \times Y$. Let $(x, y) \in F$ and suppose that $\left(x^{\prime}, y^{\prime}\right)$ is an element of $X \times Y$ for which $\left(q_{X} \times q_{Y}\right)\left(x^{\prime}, y^{\prime}\right)=$ $\left(q_{X} \times q_{Y}\right)(x, y)$. Thus $q_{X}\left(x^{\prime}\right)=q_{X}(x)$ and $q_{Y}\left(y^{\prime}\right)=q_{Y}(y)$. Since $x \in F^{-1}(y)$ and $\left(q_{X}, M_{X}\right)$ distinguishes $F^{-1}(y)$, we have $x^{\prime} \in F^{-1}(y)$, i.e. $y \in F\left(x^{\prime}\right)$. Since $\left(q_{Y}, M_{Y}\right)$ distinguishes $F\left(x^{\prime}\right)$, we have $y^{\prime} \in F\left(x^{\prime}\right)$, i.e. $\left(x^{\prime}, y^{\prime}\right) \in F$.

(ii) Suppose that $F: X \rightarrow Y$ is graph-distinguishable. By Lemma 3.6, $\{F(x): x \in X\}$ is distinguishable if $X$ is separable. Applying this lemma to $F^{-1}$ : 
$Y \rightarrow X$, we can conclude that $\left\{F^{-1}(y): y \in Y\right\}$ is distinguishable if $Y$ is separable.

Now let $X$ be compact. By symmetry, it remains only to prove that, if $Y$ is either compact or separable, then $\{F(x): x \in X\}$ is distinguishable.

Let $(q, M)$ distinguish $F \subseteq X \times Y$. Consider the function $b: Y \rightarrow C(X, M)$, defined by $b(y)=q_{y}$ where $q_{y}(x)=q(x, y)$ for each $x$ in $X$. Now $b$ is continuous and $C(X, M)$ is metrizable (see, for example, [4, pp. 261, 270\}).

If $Y$ is either compact or separable, then $b(Y)$ is a separable metrizable space.

We show now that $(b, b(Y))$ distinguishes $\{F(x): x \in X\}$. Let $y \in F(x)$, and suppose that $y^{\prime}$ is an element of $Y$ for which $b\left(y^{\prime}\right)=b(y)$ (i.e. $q_{y}=q_{y^{\prime}}$ ). Thus

$$
q\left(x, y^{\prime}\right)=q_{y},(x)=q_{y}(x)=q(x, y) .
$$

Since $(x, y) \in F$ and $(q, M)$ distinguishes $F$, we have $\left(x, y^{\prime}\right) \in F$, i.e. $y^{\prime} \epsilon$ $F(x)$.

3.8. Corollary. If $F: X \rightarrow Y$ and $G: Y \rightarrow Z$ are graph-distinguishable multivalued maps then $G \circ F: X \rightarrow Z$ is graph-distinguishable provided $Y$ is separable.

The same conclusion holds if each of $X, Y$ and $Z$ is either separable or compact.

Proof. By part (ii) of Theorem 3.7, $\{G(y): y \in Y\}$ is distinguishable. Thus $\{G \circ F(x): x \in X\}$ is distinguishable. Similarly, $\left\{F^{-1}(y): y \in Y\right\}$ is distinguishable, and so $\left\{(G \circ F)^{-1}(z): z \in Z\right\}$ is distinguishable. Thus, by part (i) of Theorem 3.7, $G \circ F$ is distinguishable.

3.9. Analytic (descriptive Borel) sets are defined as u.s.c. (disjoint u.s.c.) range-compact images of $I$, and are preserved by u.s.c. (disjoint u.s.c.) rangecompact multivalued maps [5, p. 414]. The following are analogous results for E-analytic and descriptive Borel sets.

3.10. Corollary. (i) Z-analytic (descriptive Baire) subsets of separable or compact spaces are preserved by graph-distinguishable u.s.c. (disjoint u.s.c.) range-compact multivalued maps.

(ii) Z-analytic (descriptive Baire) sets are precisely the images of $\mathbf{I}$ under graph-distinguishable u.s.c. (disjoint u.s.c.) range-compact multivalued maps.

Proof. (i) Let $A$ be a $\mathcal{Z}$-analytic (descriptive Baire) subset of $X$ with $A=$ $K(I)$ as in 1.6, and let $F: X \rightarrow Y$ be graph-distinguishable, u.s.c. (disjoint u.s.c.) and range-compact. By Theorem 3.7, part (ii), $\{F(x): x \in X\}$ is distinguishable and therefore so is $\{F[K(\mathrm{i})]: \mathrm{i} \in \mathrm{I}\}$. Since $F \circ K$ is also u.s.c. (disjoint u.s.c.) and range-compact, $F(A)=F \circ K(I)$ is $Z$-analytic (descriptive Baire) by 1.10 . 
(ii) By part (i), since $I$ is a separable descriptive Barire space, $K(I)$ is $\mathcal{Z}_{\text {-analytic }}$ (descriptive Baire) if $K$ is graph-distinguishable, range-compact and u.s.c. (disjoint u.s.c.). On the other hand, let $K(\mathrm{I})$ be a $Z$-analytic (descriptive Baire) subspace of $X$ with the representation of 1.6. Since $\{K(\mathbf{i}): \mathbf{i} \in I\}$ is distinguishable and $I$ is separable and metrizable, $K: I \rightarrow X$ is graph-distinguishable by part (i) of Theorem 3.7.

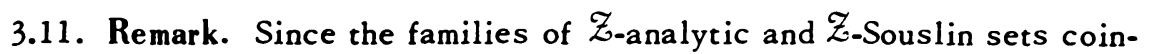
cide for compact spaces, Corollary 3.10 implies that among compact spaces, $\mathscr{Z}$. Souslin sets are preserved by continuous graph-distinguishable maps.

Corollaries 3.12 and 3.13 relate the graph-distinguishable condition to wellknown conditions.

3.12. Corollary. Continuous single-valued maps with separable metrizable ranges are graph-distinguishable. (More generally, continuous range-compact multivalued maps $F: X \rightarrow Y$, for whicb $\{F(x): x \in X\}$ is distinguishable, are graph-distinguishable.)

Proof. Let $(q, M)$ distinguish $\{F(x): x \in X\}$. We prove that $(q \circ F, \mathcal{C}(M))$ distinguishes $\left\{F^{-1}(y): y \in Y\right\}$ [and hence $F: X \rightarrow Y$ is graph-distinguishable by Theorem 3.7 (i)]. Suppose $x \in F^{-1}(y)$ and $x^{\prime}$ is an element of $X$ for which $(q \circ F)\left(x^{\prime}\right)=(q \circ F)(x)$, i.e. $q\left[F\left(x^{\prime}\right)\right]=q[F(x)]$. Since $(q, M)$ is distinguishes $\{F(x): x \in X\}$, we have $F\left(x^{\prime}\right)=F(x)$. Thus $y \in F(x)=F\left(x^{\prime}\right)$, and $x^{\prime} \in F^{-1}(y)$.

3.13. Corollary. Perfect open single-valued maps (more generally, open, closed domain-compact multivalued maps) with separable metrizable domain are graph-distinguishable.

Proof. Let $F: X \rightarrow Y$ be open, closed and domain-compact with $X$ separable and metrizable. Since $\{x: x \in X\}$ is distinguishable, so is $\{F(x): x \in X\}$ by part (iii) of Theorem 2.4. Thus, by Theorem 3.7, $F: X \rightarrow Y$ is graph-distinguishable.

3.14. Theorem 3.7 also leads to the following result, a generalization of the well-known metrization theorem (Corollary 3.17) for compact spaces.

3.15. Theorem. A compact space $Y$ is metrizable if, and only if, there exists a compact space $X$ and a graph-distinguisbable multivalued map $F: X \rightarrow$ $Y$ such that $F^{-1}\left(y_{1}\right) \neq F^{-1}\left(Y_{2}\right)$ for $y_{1} \neq y_{2}$.

Proof. Let $(q, M)$ distinguish $F$. The map $b: Y \rightarrow C(X, M)$, defined by $b(y)=q_{y}$ and $q_{y}(x)=q(x, y)$, distinguishes $\{F(x): x \in X\}$ (see the proof of Theorem 3.7). Here, $b$ is one to one, for if $y_{1} \neq y_{2}$, there exists an element $x$ of $X$ such that $F(x)$ contains exactly one element of $\left\{y_{1}, y_{2}\right\}$. Since 


$$
b[F(x)] \cap b[Y \sim F(x)]=\varnothing,
$$

we have $b\left(y_{1}\right) \neq b\left(y_{2}\right)$. Hence $b$ is a homeomorphism, and $Y$ is metrizable.

If $Y$ is compact and metrizable, then $\Delta=\{(y, y): y \in Y\}$ is distinguishable in $Y \times Y$ and $\Delta^{-1}\left(y_{1}\right) \neq \Delta^{-1}\left(y_{2}\right)$ for $y_{1} \neq y_{2}$.

3.16. Remark. The above proof illustrates homeomorphisms between every compact metrizable space $Y$ and compact subspaces of $C^{*}(Y)$.

3.17. Corollary (see Sneider [8]). A compact space $Y$ is metrizable if, and only if, it has a $\mathfrak{S}_{\delta}$ diagonal.

Proof. Since the diagonal $\Delta$ is a zero set of $Y \times Y$, it is distinguishable. Also $\Delta^{-1}\left(y_{1}\right) \neq \Delta^{-1}\left(y_{2}\right)$ for $y_{1} \neq y_{2}$, and so the previous corollary applies.

\section{REFERENCES}

1. P. S. Alexandroff and P. S. Urysohn, Mémoire sur les espaces topologiques compacts, Verh. Kon. Akad. Wetensch. Amsterdam. Afd. Natuurk. (1) 14 (1929), no. 1, 1-96; Russian rev. transl., On compact topological spaces, Trudy Mat. Inst. Steklov. 31 (1950). MR 13, 264.

2. E. J. Braude, Descriptive Baire and descriptive Z-analytic sets, Proc. London Math. Soc. (3) 23 (1971), 409-427.

3. - The projection of a compact zeroset is a zeroset, Notices Amer. Math. Soc. 18 (1971), 783. Abstract \#678-54-2.

4. J. Dugundji, Topology, Allyn and Bacon, Boston, Mass., 1966. MR 33 \#1824.

5. Z. Frolik, A survey of separable descriptive theory of sets and spaces, Czechoslovak Math. J. 20 (95) (1970), 406-467. MR $42 \# 1660$.

6. J. E. Jayne, Descriptive set theory in compact spaces, Notices Amer. Math. Soc. 17 (1970), 268. Abstract \#672-642.

7. J. L. Kelley, General topology, Van Nostrand, Princeton, N. J., 1955. MR 39 \#07.

8. V. E. Šneider, Continuous images of Suslin and Borel sets: Metrization theorems, Dokl. Akad. Nauk SSSR 50 (1945), 77-79. (Russian) MR 14, 782.

DEPARTMENT OF MATHEMATICS, SETON HALL UNIVERSITY, SOUTH ORANGE, NEW JERSEY 07079 